\title{
Smoking in migrants in New South Wales, Australia: Report on data from over 100000 participants in the 45 and Up Study
}

\author{
MARIANNE F. WEBER ${ }^{1}$, EMILY BANKS $^{2} \&$ FREDDY SITAS $^{1}$ \\ ${ }^{1}$ Cancer Research Division, Cancer Council NSW, Sydney, Australia, and ${ }^{2}$ National Centre for Epidemiology and \\ Population Health, The Australian National University, Canberra, Australia
}

\begin{abstract}
Introduction and Aims. Approximately 25\% of the Australian population was born abroad, yet there has been very little tobacco control aimed at culturally and linguistically diverse communities and limited data exist on smoking among Australian migrants. The aim of this study was to compare smoking characteristics of Australian migrants (in terms of place of birth and age migrated) to those of Australian-born residents. Design and Methods. A cross-sectional analysis of self-reported questionnaire data from 53207 women and 48777 men aged 45 years or over in The 45 and Up Study in Australia (2006-2008) was performed. Results. 52.6\% (95\% confidence intervals 52.1-53.0) of men and 35.5\% (35.1-35.9) of women reported ever being a regular smoker and 7.6\% (7.4-7.8) and 7.3\% (7.1-7.5) reported current smoking, respectively. Compared with Australian-born men, a higher proportion of men born in Europe, North Africa and the Middle East were current smokers, with odds ratios adjusted for age, income, education and place of residence (OR; 95\% confidence intervals) ranging from 1.30 (1.16-1.45) to 1.96 (1.49-2.58). Compared with Australian-born women, a lower proportion of women from East $(0.21 ; 0.12-0.36)$ and Southeast Asia $(0.38 ; 0.26-0.54)$ were current smokers and a higher proportion of women from New Zealand $(1.45 ; 1.17-1.79)$ and the UK/Ireland $(1.25 ; 1.12-1.40)$ were current smokers. Among women born in Asia, the risk of smoking increased significantly the younger they migrated to Australia. Duration smoked and amount smoked per day were primarily lower among migrants than Australian-born. Conclusions. Smoking prevalence varies substantially across cultural subgroups. Understanding smoking dynamics across diverse cultural groups will assist in better targeting of tobacco control programs. [Weber WF, Banks E, Sitas F. Smoking in migrants in New South Wales, Australia: Report on data from over 100000 participants in the 45 and Up Study. Drug Alcohol Rev 2010]
\end{abstract}

Key words: tobacco smoking, migrant, Australia.

\section{Introduction}

Australia has had an impressive history of tobacco control, such that current smoking rates in ages 14 years and over are approximately $16.6 \%$ and declining use has been observed across all social classes and ages. Concerted efforts have been made to restrict the sale of tobacco products, ban advertising, and increase taxation to the extent that Australia is now regarded by British American Tobacco as 'one of the darkest markets in the world' $[1,2]$. Despite these declines in tobacco prevalence, tobacco persists as the leading health problem in Australia, being responsible for 15\% of total deaths [3], $20 \%$ of deaths in Aboriginal people
[4] and is possibly responsible for a similar magnitude of burden in other marginalised groups. While much work needs to be done to sustain and improve the current levels of achievement in tobacco control, very little data exist on smoking among Australian migrant groups. Compared with English-speaking communities, there is a relative dearth of tobacco control activity in culturally and linguistically diverse communities in Australia.

Australia is a highly heterogeneous country with approximately $25 \%$ (5.3 million) of its current population born abroad from around 200 nationalities [5]. Approximately $62 \%$ of migrants originate from countries where English is not the main spoken language

Marianne F. Weber BA (Hons), PhD, Research Fellow, Emily Banks B Med Sci (Hons), MB BS (Hons), PhD, NHMRC Senior Research Fellow, Freddy Sitas BSci, MSc(MED), MSC Epidemiology, DPhil Epidemiology, Director, Cancer Research Division, Cancer Council NSW. Correspondence to Dr Marianne F. Weber, Cancer Research Division, Cancer Council NSW PO Box 572, Kings Cross 1340, Australia. Tel: +61 2 93341415; Fax: +61 2 83023550; E-mail: mariannew@nswcc.org.au

Received 12 August 2009; accepted for publication 18 August 2010. 
and $16 \%$ of Australians speak a language other than English at home, with 3\% not proficient in spoken English [6]. Understanding smoking dynamics across such diverse groups would assist in better targeting of tobacco control programs in Australia. In a national survey conducted in 2007 it was found that among Australian people whose main spoken language at home was not English, the proportion of smokers was lower than English-only speakers [2]. However, these statistics can be misleading because: (i) they do not take sociodemographic factors into account, which are strongly associated with smoking; and (ii) lifestyle characteristics across migrant groups are not uniform. One report from the National Health Survey in 1995 found that after adjusting for age and socioeconomic status, men born in parts of Europe and the Middle East were more likely to smoke than men born in Australia/New Zealand and women born in UK/Ireland were more likely to smoke than those born locally [7]. In contrast, men and women born in Asia were less likely to smoke than Australian/New Zealand born and women born in parts of Europe and the Middle East were also less likely to smoke than those born locally. In the 14 years since that report, smoking rates in Australia have markedly decreased; however, tobacco control efforts in culturally and linguistically diverse communities have been less intense, which means that the gap in smoking rates between Australian-born and immigrants may have changed substantially.

We used cross-sectional data from the largest cohort study in Australia, the 45 and Up Study, to compare smoking characteristics of Australian migrants (in terms of place of birth) to those of Australian-born residents in individuals aged 45 years and over. This age group has a high proportion of ex-smokers [2] and includes the ages with the greatest smoking-related morbidity and mortality; it is of interest to determine which groups continue to smoke in the face of the rapidly increasing risks with age.

\section{Methods}

\section{Study population}

The 45 and Up Study is a population-based cohort study of people aged 45 years and over resident in New South Wales, Australia [8]. The population of New South Wales comprises $32.6 \%$ of the total Australian population (7 million in 2008) [9]. Participants are randomly sampled from Medicare Australia (Australia's public health insurance system, which covers, in part, the health-care costs of all citizens and permanent residents of Australia, some temporary residents and refugees). Residents in regional and remote areas and those aged 80 years and over were oversampled by a factor of two. Participants completed a mailed selfadministered questionnaire and consent form (in English; available at http://www.45andUp.org.au). A small number of participants $(<1 \%)$ joined the study by contacting the coordinating centre directly and volunteering. This cross-sectional analysis uses questionnaire data from 103042 people who returned their consent forms and questionnaires between the inception of the study in February 2006 and July 2008. In order to provide sufficient sample sizes for robust comparative work, place of birth was grouped into broad regions according to a modified version of the Global Burden of Disease Study (see Table 1).

The 45 and Up Study has been approved by the University of New South Wales Human Research Ethics Committee. Additional ethical approval for this specific project was also provided by the Cancer Council New South Wales Ethics Committee.

\section{Ascertainment of smoking history}

Self-reported history of smoking was ascertained from the question, 'Have you ever been a regular smoker?' If 'Yes', how old were you when you started smoking regularly? Are you a smoker now? If not, how old were you when you stopped smoking regularly? Never smokers were participants who answered 'No' to the question, 'Have you ever been a regular smoker?'. Amount of tobacco smoked per day was ascertained from the question, 'About how much do you/did you smoke on average each day?' (we assumed weights of $1 \mathrm{~g}$ per cigarette, $1 \mathrm{~g}$ per cigar and a conservative $1 \mathrm{~g}$ per pipeful).

\section{Analyses}

We examined the distribution of current smokers by place of birth, age since migration and years lived in Australia. Odds ratios and $95 \%$ confidence intervals (CI) of smoking by migrant factors were estimated using logistic regression. Each model was stratified by gender and adjusted for age (4 levels: 45-54; 55-64; $65-74 ; 75+$ ), highest level of education achieved (none; 10 years of schooling - 'School Certificate'; 12 years of schooling - 'Higher School Certificate'/trade/diploma; university degree), income (total annual household income from all sources: \$0-\$19 999; \$20 000$\$ 49$ 999; \$50 000+), and place of residence (major city; inner region; outer region/remote/very remote). The effect of age migrated to, and years lived in, Australia on smoking rates were each analysed as a continuous variable in separate analyses for both men and women, grouped by broad regional area: Asia (South, Central, Southeast, East), Continental Europe and the Middle East/North Africa (Central, East and West Europe, 


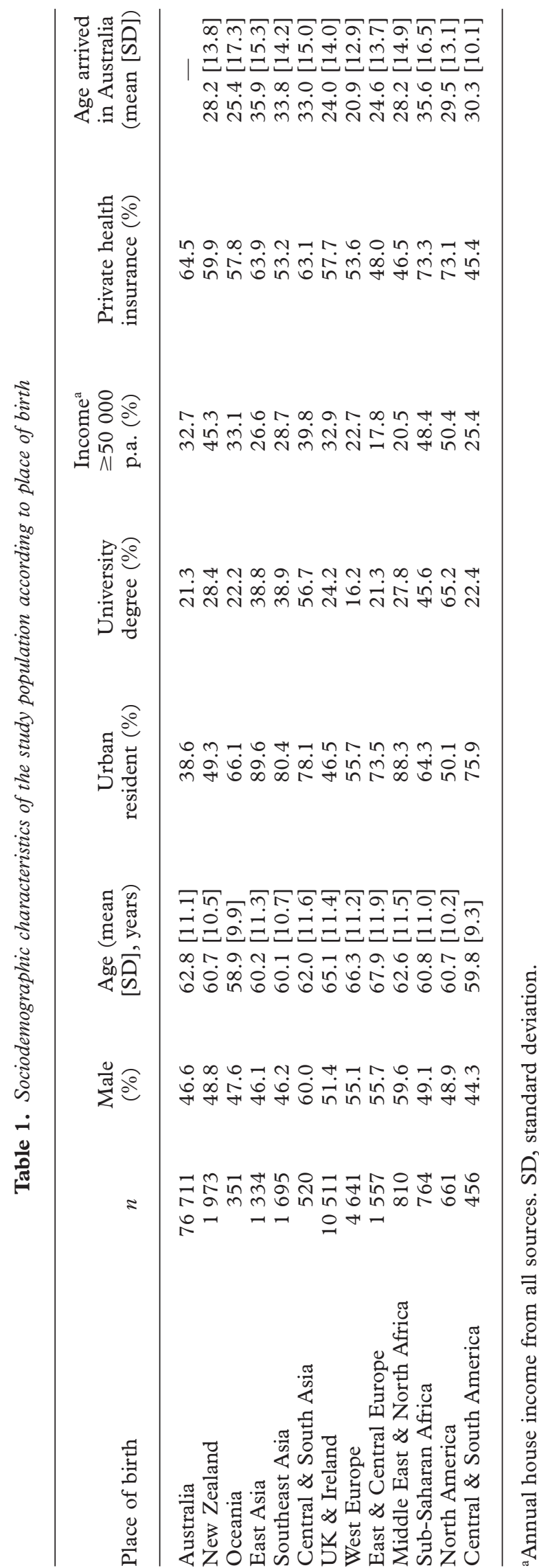

Middle East, North Africa) and English-speaking countries (UK, Ireland, New Zealand, North America). The effect of age of migration and years lived in Australia on smoking rates were also analysed as categorical variables $(0-10 ; 11-20 ; 21-30 ; 31-40 ; 40+$ years $)$ and compared with Australian-born. All models included a 'missing/ unknown' level for each covariate (data not shown).

The mean number of years smoked and amount of tobacco smoked per day for 'current smokers' were estimated from a linear regression of 'number of years smoked' or 'amount of tobacco smoked/day' on 'age' and 'place of birth'. Values are presented (in Tables 2,3) as the mean number of years smoked and mean amount of tobacco smoked/day for each place of birth at age 63 years (mean age of the sample). While the mean number of years smoked increases with age, mean differences between places of birth remain constant across age.

A total of 1038 (1\%) participants did not adequately describe a place of birth and were excluded from all analyses. The $20(0.02 \%)$ participants who were missing data on smoking status were also excluded, leaving 101984 participants for the main analyses.

\section{Results}

Of the total 101984 participants, 76711 (75.2\%) were born in Australia. The remainder reported 162 places of birth, the leading ones being UK/Ireland (10 511), New Zealand (1973), Germany (1092), the Netherlands (1030), China/Taiwan/Hong Kong/Macau (1037) and Italy (817). Table 1 shows the sociodemographic breakdown of participants. Compared with Australian-born individuals, people from Europe tended to be older, whereas those born in America, Asia, sub-Saharan Africa and Oceania were younger. In general, migrants had higher educational levels than Australian-born, tended to live in large urban centres and reported lower household incomes. A greater proportion of migrants than Australian-born did not have private health insurance. On average, participants from Asia and sub-Saharan Africa migrated to Australia when they were in their 30's, whereas participants from Europe, the Middle East/North Africa and New Zealand migrated when they were in their 20's.

The overall prevalence of smoking within the cohort, weighted for age and region of residence using the distribution of the NSW population by age according to the Accessibility/Remoteness Index of Australia (ARIA+ [10]; 2006) [11], was $8.4 \%$ for men and $6.7 \%$ for women. Tables 2,3 show crude smoking prevalences for men and women in the cohort, respectively, by place of birth, as well as smoking duration and amounts for current smokers adjusted for age. For each place of birth, men had a higher mean duration of smoking than women. Among smokers, men smoked more tobacco 

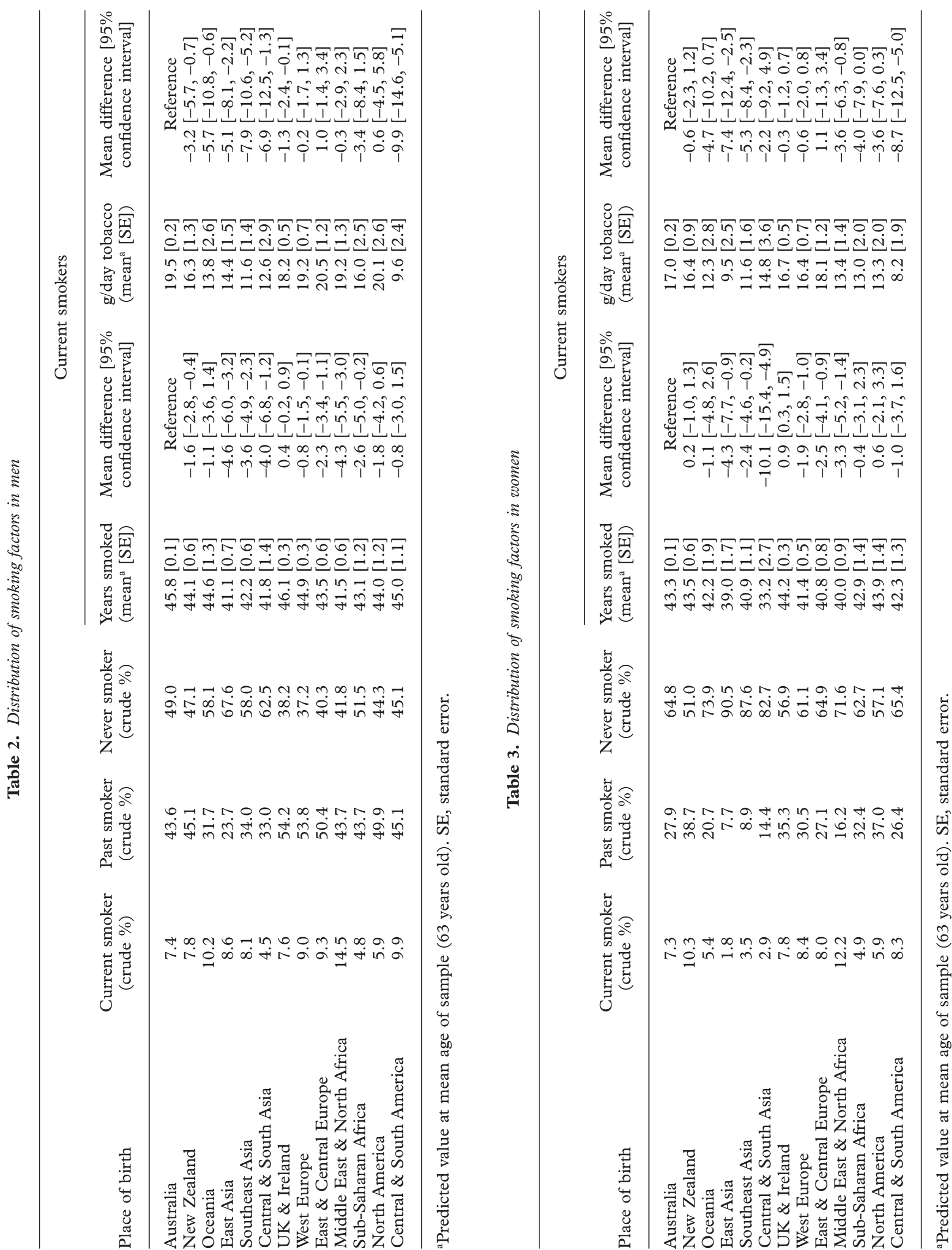
per day than women across all places of birth except Central and Southern Asia and New Zealand. After accounting for age, the mean number of years smoked was higher for Australian-born participants than all migrants groups except migrants from UK/Ireland and women from New Zealand and North America. The mean amount of tobacco smoked per day among smokers was higher for Australian-born participants than all migrant groups except those from East and Central Europe and women from North America.

After adjusting for age, education, income and place of residence, there was significant variation in the proportion of current smokers across migrant groups (Figure 1). A greater proportion of men born in Continental Western Europe $(\mathrm{OR}=1.46,95 \% \mathrm{CI}=1.26-$ 1.70), Central/Eastern Europe (1.55, 1.21-1.97), North Africa/the Middle East (1.96, 1.49-2.58) and the UK/Ireland (1.30, 1.16-1.45) reported current smoking than Australian-born men. Women born in New Zealand $(1.45,1.17-1.79)$ and the UK/Ireland $(1.25,1.12-1.40)$ were more likely to be current smokers than Australian-born women. In contrast, women born in East Asia (0.21, 0.12-0.36) and Southeast Asia (0.38, 0.26-0.54) had significantly lower adjusted odds ratios of current smoking than Australian-born women. We also examined selected non-English-speaking countries with large sample sizes. Men from the Netherlands reported higher rates of smoking than Australian-born men (1.57, 1.16-2.11), whereas there were no significant differences between men from Australia and those from Germany, China/ Taiwan/Hong Kong/Macau or Italy. Women from Germany reported higher rates of smoking than
Australian-born women (1.54, 1.16-2.06) and women from China/Taiwan/Hong Kong/Macau reported lower rates of smoking $(0.06,0.02-0.26)$. There were no significant differences in current smoking rates for women born in the Netherlands or Italy compared with Australian-born.

Figure 2 shows the odds ratios (+95\% CI) of smoking by age migrated to Australia for participants born in: English-speaking countries (UK, Ireland, New Zealand, North America, South Africa); Continental Europe, North Africa and the Middle East; and Asia. Smoking rates among women from Asia who migrated at age 0-10 years were similar to Australian-born rates. However, the odds of Asian-born women being a smoker decreased by $36 \%$ with every 10 year increase in the age they migrated to Australia (0.64, 0.51-0.81, $P=0.0002)$. A similar gradient was not apparent for Asian-born men (1.02, 0.88-1.19, $P=0.80)$. A different trend was observed for women from Englishspeaking countries, where those who migrated at a younger age had a higher rate of smoking than Australian-born women and with every 10 year increase in age migrated, the odds of being a smoker decreased by $9 \%(0.91,0.84-0.98, P=0.01)$. There was no effect of age of migration on smoking rates for men from English-speaking countries when age of migration was analysed as a continuous variable $(0.99,0.92-1.06$, $P=0.73$ ); however, those who migrated later in life had higher rates of smoking than Australian-born participants (see Figure 2). A similar pattern was observed in relation to time since migration, which illustrates the close correspondence in smoking behaviour between age at and time since migration (Figure 3).

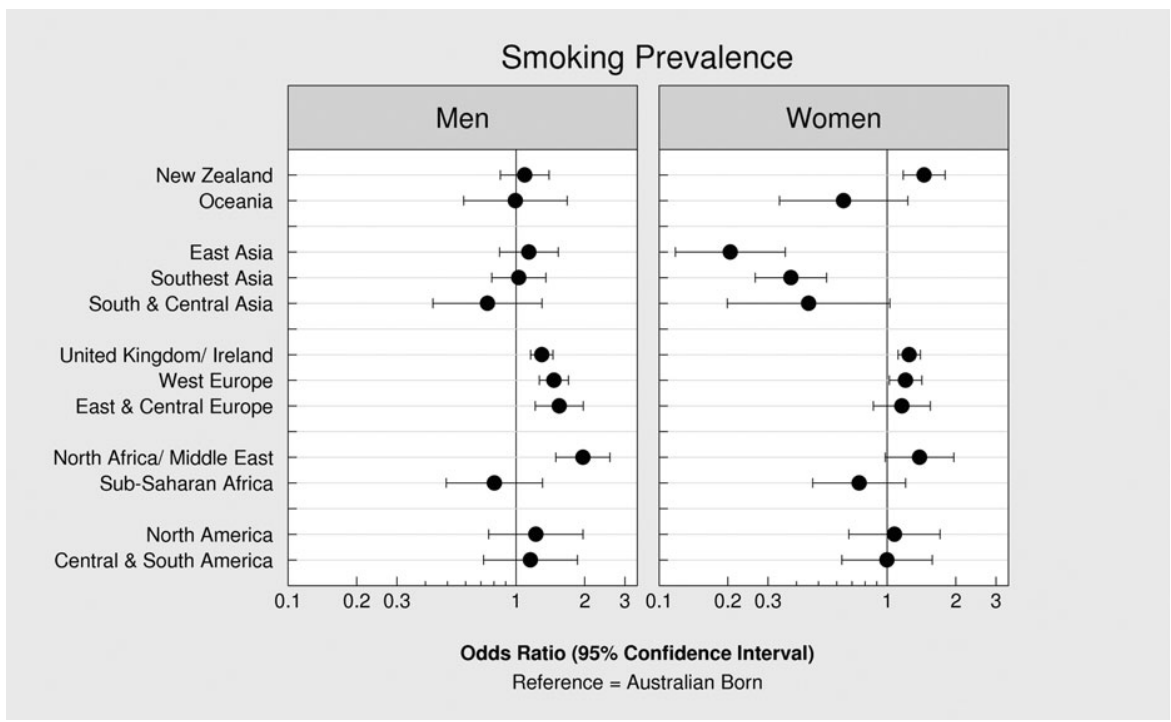

Figure 1. Odds ratio of being a current regular smoker among male and female migrants compared with Australian-born participants. Odds ratios are adjusted for age, education, income and region of residence. 


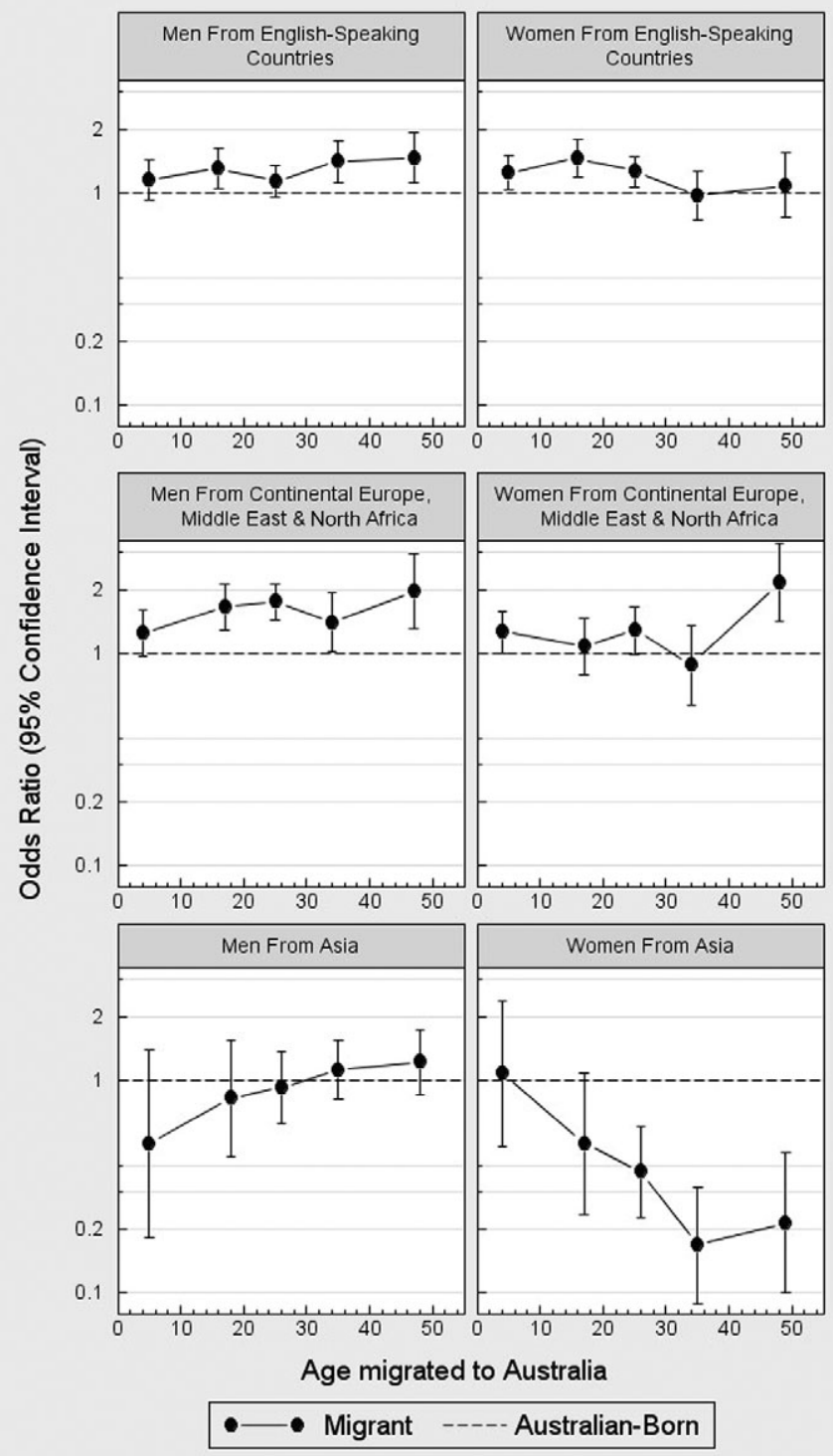

Figure 2. Odds ratio of being a current regular smoker among male and female migrants from: English-speaking countries; continental Europe, North Africa and the Middle East; and Asia by age migrated to Australia compared with Australian-born participants. Odds ratios are plotted at the median value for each category of age migrated: 0-10 years, 11-20 years, 21-30 years, 31-40 years, and 40+ years, and are adjusted for age, education, income and region of residence.

Men from continental Europe, North Africa and the Middle East had a higher rate of smoking than Australian-born men regardless of the age they migrated to Australia, with no gradient in smoking rates with age migrated $(1.00,0.90-1.10, P=0.95)$. There was also no apparent gradient in smoking rates by age of migration for women from continental Europe, North Africa and the Middle East (1.01, 0.91-1.12, $P=0.88$ ); however, women who migrated from this region after the age of 40 years had twice the odds of being a current smoker than Australian-born participants $(\mathrm{OR}=2.15,1.42-3.26$; Figure 2).

\section{Discussion}

These data show that smoking rates among migrant groups in the Australian population are not uniform and highlight the possibility that although the overall prevalence of smoking in the community is decreasing, there may be pockets of the population that retain high rates of smoking. After adjusting for age, education, place of residence and income, smoking rates among many migrant groups, and in particular migrants from non-English-speaking places, were significantly different from the rate of Australian-born. Specifically, men 


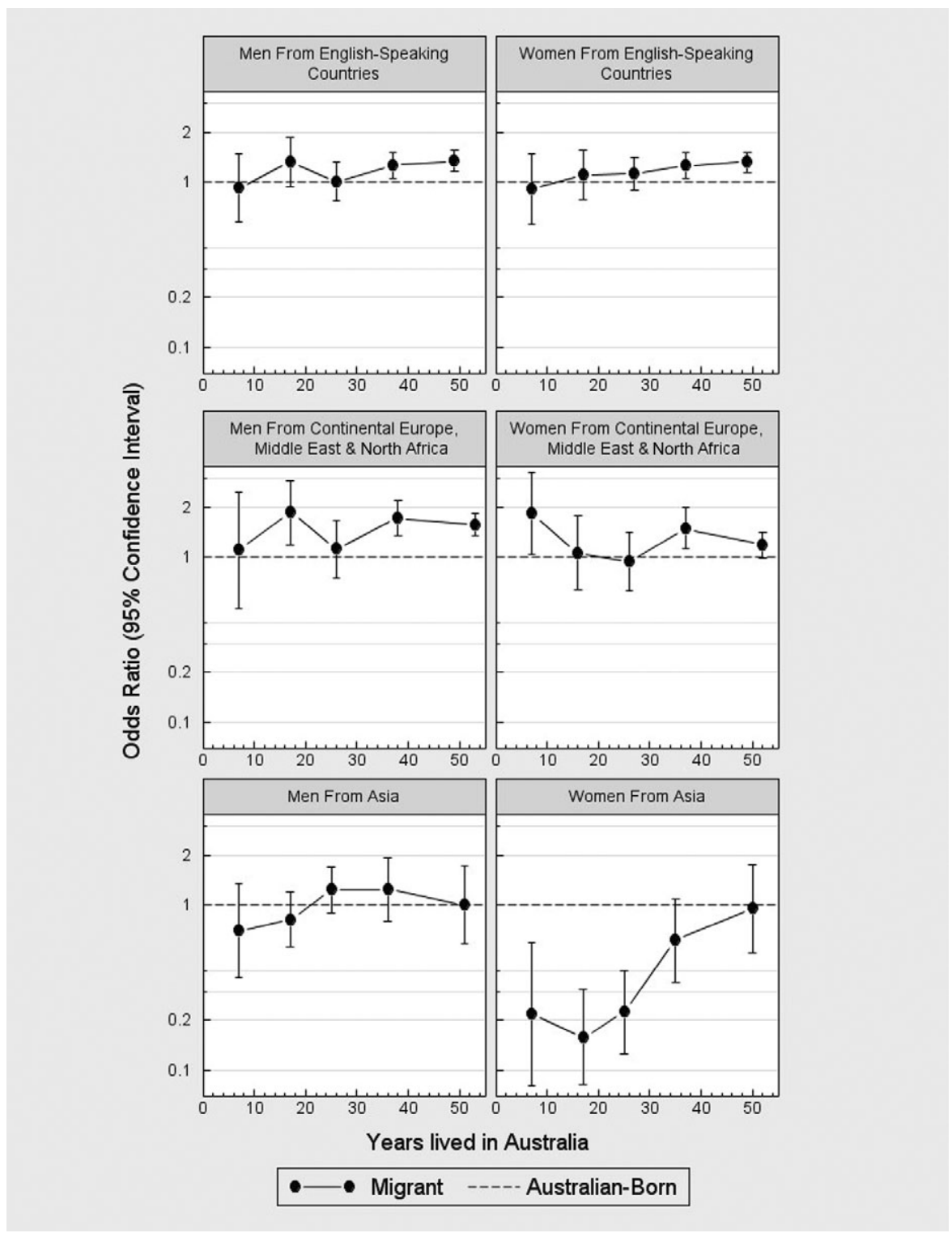

Figure 3. Odds ratio of being a current regular smoker among men and women migrants from: English-speaking countries; continental Europe, North Africa and the Middle East; and Asia by years lived in Australia compared with Australian-born participants. Odds ratios are plotted at the median value for each category of years lived in Australia: 0-10 years, 11-20 years, 21-30 years, 31-40 years, and 40+ years, and are adjusted for age, education, income and region of residence.

born in Europe, North Africa and the Middle East were more likely to smoke than men born in Australia, and women born in Asia were less likely to smoke than Australian-born women. Women from some Englishspeaking places (New Zealand, the UK and Ireland) were significantly more likely to smoke than Australianborn women. The age at which migrants arrived in Australia affected the rate of smoking most markedly for women born in Asia. That is, the younger they arrived, the more similar their rate of smoking to Australian-born participants. Current smokers born outside of Australia tended to smoke less tobacco per day than Australian-born smokers and had not smoked for as long as Australian-born smokers (taking age into account). For example, men from Southeast Asia had similar rates of smoking to Australian-born participants, but they smoked a mean of $7.9 \mathrm{~g}$ less tobacco per day.

Focusing prevention programs in non-mainstream language groups is an international and local challenge. While a comprehensive set of controls are evolving to reduce tobacco consumption in Australia, and many control measures such as taxation and legislation are not language-dependent, those measures involving 
information and support are largely aimed at Englishspeaking communities. A recent government document on tobacco control in Australia reported that such tobacco prevention measures in culturally and linguistically diverse communities had been minimal and that people from non-English-speaking backgrounds have had much more limited access to information and support, especially in regional areas [12]. Our data highlight the need to target tobacco prevention and support programs at men from Europe and especially men from the Middle East/North Africa. Interestingly, our data are very similar to the report from the National Health Survey 10 years prior to this study [7]. Notable differences between the two studies, however, are that the survey 10 years ago reported that men from Asia were less likely to smoke than Australian-born $(\mathrm{OR}=0.68)$, as were women from the Middle East $(\mathrm{OR}=0.61)$. Our analyses found no statistical differences in smoking for these groups compared with Australian-born individuals. Although our sample may not be representative, and therefore null results should be interpreted with caution, the point estimates in our study were close to 1 for Asian-born men, and greater than 1 for Middle-Eastern/North African women. If we compared the two surveys directly, it appears that over the last 10 years, smoking rates in these groups are becoming more like those observed in Australian-born. This indicates that perhaps these migrant groups are either being left behind in terms of tobacco control (because of language and cultural barriers) or that the cultural norms surrounding smoking in these groups are changing. A more comprehensive survey of smoking in these groups is needed to determine if this effect is real and if so, why. There may be a similar need to target prevention programs at women from Asia because the results from our study suggest that smoking rates are significantly lower than Australian-born women, but that the rate increases towards Australian-born the younger they migrate to Australia. These trends are also illustrated in relation to time since migration in Figure 3. Assuming age of arrival or time since arriving in a host country are indirect proxies of acculturation, then stronger tendencies of acculturation are more evident in women from Asia (but not in Asian men, or any other group of men or women, who seem to have retained the same rates of smoking as Australian-born irrespective of their age migrating to Australia). Clearly, more work is needed to unravel this complex picture.

Our report found that after adjusting for sociodemographic variables, women from the UK/Ireland and New Zealand were more likely to be current smokers than Australian-born women. A similar finding was reported in the 1995 National Health Survey analysis, where women from the UK/Ireland were more likely to smoke than Australian/New Zealand women [7].
It is unclear why these groups of women have been more resistant to tobacco control mechanisms than Australian-born women, but it appears that this effect has remained stable over the last 10 years, at least for women born in the UK/Ireland. Overall, smoking prevalence among women in the UK/Ireland and in New Zealand is slightly higher than in Australia [13] so it appears that British, Irish and New Zealand women migrants in Australia hold on to the smoking norms of their home country, even though they are exposed to Australian tobacco control measures.

The 45 and Up Study is a cohort study, requiring a long-term commitment from participants, and is not designed to be representative of the general population, nor is it suitable for estimating the point prevalence of smoking. Cohort study participants tend to be healthier and more health conscious than non-participants [14]; hence, we expected smoking rates to be somewhat lower in the 45 and Up Study cohort than the general population. Indeed, the adjusted smoking rates within this cohort $(8.4 \%$ for men and $6.7 \%$ for women) were less than that estimated in people aged 45 years and over in the NSW population in 2007 (12\% - although, this rate also included 'occasional smokers') [15]. Although the cohort includes participants with the wide range of incomes necessary for internal comparisons [8], including those with low incomes, disadvantaged individuals may be less likely to take part. Cohort studies are designed to provide reliable information on the effects of different risk factors on outcomes, rather than population prevalence estimates and are generalisable even when based on highly selected groups $[16,17]$. Moreover, relative risk estimates from the 45 and Up Study (response rate 18\%) do not differ materially from those calculated using data from the more representative NSW Health Survey (response rate $\sim 62 \%$ ), when comparable variables are used; this includes variables relating to smoking [18].

The proportion of migrants in the cohort with English as a second language was smaller than the proportion in the NSW population [11] and this, along with the fact that the questionnaire was available only in English, suggests reduced participation among nonEnglish-speaking migrants. Migrants with limited skills in English may be more likely than other participants to misunderstand the questionnaire, leading to greater measurement error. These issues mean that the odds ratios associated with smoking may be underestimated. Potential bias resulting from the 'healthy cohort' effect, if it is present, and reduced participation from more disadvantaged and less acculturated migrants, would lead to more conservative results. Caution must therefore be exercised when interpreting negative results. However, even within this selective group we observed marked differences in smoking rates between migrant 
groups and Australian-born participants and these results were somewhat similar to the report from the National Health Survey in 1995 [7].

Generalisations about smoking in local migrant communities may distort perceptions and local needs of prevention programs. This is because generalisations from lifestyle surveys conducted in respective places of birth may not pertain to lifestyle characteristics of migrants living in Australia. One reason for this is that the demographic profile of many migrant groups differs from the demographic profile of their home country. Migration policies to Australia are complex, change over time and vary from a selection of skilled migrants using a points system to small groups of refugees accepted on humanitarian grounds [19]. As such, socioeconomic factors among migrant groups, such as educational attainment, can be higher than that of their home country (and Australian-born for that matter) [6]. Further, smoking rates are dynamic and change over time both within the country of origin and locally. Thus, understanding smoking dynamics across such diverse groups will assist in better targeting of tobacco control programs in Australia. As the 45 and Up Study matures we will be able to document smoking-related morbidity in these migrant groups in the coming years.

\section{Acknowledgements}

The 45 and Up Study is managed by the Sax Institute in collaboration with major partner: Cancer Council New South Wales and partners the New South Wales Division of the National Heart Foundation of Australia; the New South Wales Department of Health; beyondblue the national depression initiative; the New South Wales Department of Ageing, Disability and Home Care and Uniting Care Ageing.

\section{References}

[1] Scollo MM, Winstanley MH. Tobacco in Australia: facts and issues, 3rd edn. Melbourne: Cancer Council Victoria, 2008.

[2] Australian Institute of Health and Welfare. 2007 national drug strategy household survey: detailed findings. Canberra: AIHW, 2008.
[3] Begg SJ, Vos T, Barker B, Stanley L, Lopez AD. Burden of disease and injury in Australia 2003. Canberra: AIHW, 2007.

[4] Vos T, Barker B, Stanley L, Lopez AD. The burden of disease and injury in Aboriginal and Torres Strait Islander peoples 2003. Brisbane: School of Population Health, The University of Queensland, 2007.

[5] Australian Bureau of Statistics. Migration 2006-2007. Canberra: Commonwealth of Australia, 2008.

[6] Australian Bureau of Statistics. Migrants, census of population and housing, 2006. Canberra: Commonwealth of Australia, 2007.

[7] Siahpush M, Borland R. Socio-demographic variations in smoking status among Australians aged $>$ or $=18$ : multivariate results from the 1995 National Health Survey. Aust N Z J Public Health 2001;25:438-42.

[8] Banks E, Redman S, Jorm L, et al. Cohort profile: the 45 and up study. Int J Epidemiol 2008;37:941-7.

[9] Australian Bureau of Statistics. Australian demographic statistics, Jun 2008. Canberra: Commonwealth of Australia, 2008.

[10] Glover J, Tennant S. Remote areas statistical geography in Australia: notes on the accessibility/remoteness index for australia (ARIA+ version). Adelaide: Public Health Information Development Unit, University of Adelaide, 2002.

[11] Australian Bureau of Statistics. Census of population and housing. Canberra: Commonwealth of Australia, 2006.

[12] Tobacco Working Group. Technical report No 2. Tobacco in Australia: making smoking history. Canberra: National Preventative Health Taskforce, 2008.

[13] Mackay J, Eriksen M. The tobacco atlas. Brighton: World Health Organisation, 2002.

[14] Breslow NE, Day N. Statistical methods in cancer research, Vol. II. The design and analysis of cohort studies. IARC Scientific Publication No 82. Lyon: International Agency for Research on Cancer, 1987.

[15] Centre for Epidemiology and Research. 2007 report on adult health from the New South Wales population health survey. Sydney: NSW Department of Health, 2008.

[16] Doll R, Peto R, Boreham J, Sutherland I. Mortality in relation to smoking: 50 years' observations on male British doctors. BMJ 2004;328:1519.

[17] Willett WC, Blot WJ, Colditz GA, Folsom AR, Henderson $\mathrm{BE}$, Stampfer MJ. Merging and emerging cohorts: not worth the wait. Nature 2007;445:257-8.

[18] Mealing N, Banks E, Jorm L, Steel D, Clements M, Rogers $\mathrm{K}$. Investigation of relative risk estimates from studies of the same population with contrasting response rates and designs. BMC Med Res Methodol 2010;10:26.

[19] Statistics Section Department of Immigration and Multicultural Affairs. Immigration: federation to century's end 1901-2000. Canberra: Commonwealth of Australia, 2001. 